\title{
Extensive practice improves adaptation to predictable perturbations in a sequential coincident timing task
}

\author{
Herbert Ugrinowitsch ${ }^{1,}$, Rodolfo Novellino Benda ${ }^{2}$, Umberto Cesar Corrêa $^{3}$, Go Tani $^{3}$ \\ ${ }^{1}$ Sports Department, Universidade Federal de Minas Gerais, Belo Horizonte, Brazil \\ ${ }^{2}$ Physical Education Department, Universidade Federal de Minas Gerais, Belo Horizonte, Brazil \\ ${ }^{3}$ Pedagogy Department, Universidade de São Paulo, São Paulo, Brazil
}

\section{Email address:}

herbertu@ufmg.br (H. Ugrinowitsch), rodolfobenda@yahoo.com.br (R. N. Benda), umbertoc@usp.br (U. C. Corrêa), gotani@usp.br (G. Tani)

\section{To cite this article:}

Herbert Ugrinowitsch, Rodolfo Novellino Benda, Umberto Cesar Corrêa, Go Tani. Extensive Practice Improves Adaptation to Predictable Perturbations in a Sequential Coincidente Timing Task. American Journal of Life Sciences. Vol. 2, No. 2, 2014, pp. 90-95.

doi: 10.11648/j.ajls.20140202.19

\begin{abstract}
The levels of learning in motor tasks have been investigated by means of extensive practice (i.e., practice that is continued beyond the achievement of performance stabilization), which shows better performance than practice until performance stabilization when facing situations that require adaptation. However, the better performance of extensive practice has been tested with unpredictable perturbation, in which changes are necessary after the movement onset, but not with predictable perturbation, which allows planning a new organization of the action before the movement onset. The present study investigated adaptation to predictable perturbation, comparing no performance stabilization at all, practice until performance stabilization and practice beyond performance stabilization, i.e., extensive practice, in a coincident timing task. This task required the performance of a sequence of movements in accordance with a visual stimulus. Forty-five self-reported right-handed volunteers participated in this study, and they were randomly divided into three groups during the first phase of the study: Pre-Stabilization (PG), Stabilization (SG) and Extensive Practice (EG), which were operationally defined as 10 trials, three trials in a row with absolute error $(\mathrm{AE}) \leq 30 \mathrm{msec}$ and six blocks of three trials in a row with $\mathrm{AE} \leq 30 \mathrm{msec}$, respectively. In the second phase, the velocity of the visual stimulus changed, causing a perceptual perturbation. The results showed that adaptation is easier after performance stabilization and that the variability observed after performance stabilization could be a source of adaptability. In general, these results indicate that the process of motor learning continues beyond performance stabilization.
\end{abstract}

Keywords: Adaptation, Extensive Practice, Stabilization, Predictable Perturbation

\section{Introduction}

The acquisition of motor skills requires practice [1]. In the last few years, studies have shown that extensive practice (i.e., practice beyond performance stabilization) provides individuals with increased capacity to adapt to new situations [2,3]. Thus, motor learning can be conceived of as a continuous process [4] that extends beyond performance stabilization [5]. For instance, with extensive practice, individuals become able to adopt different strategies to reach the goal of the task [6]. It has been suggested that extensive practice might change the structure that controls the skill and that perturbations may expose different levels of learning, the highest of which is the ability to adapt to these perturbations $[2,3,7]$. A perturbation refers to a change in the task (e.g., a new goal), which requires a change in performance, that is, adaptation [2].

Adaptation has been found to differ depending on the predictability of the perturbation [8]. For instance, Fonseca et al. [2] have shown that extensive practice with a sequential coincident timing task increases the ability to adapt to an unpredictable perturbation compared with practice until performance stabilization. Extensive practice increased the variability of the structure that controls the skill, thereby providing individuals with the ability to adapt to unpredictable perturbation. This type of perturbation requires changes in the planned action after the movement onset, which demands that the structure that controls the motor skill must be highly adaptable [9]. Extensive practice improves the ability to adapt by increasing the variability, 
particularly of the last component of the sequential task [2].

It has also been suggested that adaptation is facilitated when some type of cue is provided to the participant prior to the trial (making the perturbation predictable) compared with no cues at all [9]. It is likely that the cue enables participants to plan changes in the action before the movement begins. Therefore, we sought to investigate the effects of planning an action in advance [10] using an initially unpredictable, abrupt perturbation that demands a large change in the response and that is introduced and then kept constant through several trials. This condition allows the participant, after the first trial introducing the perturbation, to plan all of the changes in action in advance and to use feedback from one trial to the next to make corrections. In this situation, extensive practice may not provide an advantage in relation to practice until performance stabilization. Therefore, we sought to investigate whether the effects of extensive practice on adaptation to unpredictable perturbation are replicated with predictable perturbation.

Our expectations were as follows: first, under predictable perturbation, extensive practice would produce similar performance as practice until performance stabilization; second, extensive practice would diminish the performance variability under the perturbation condition; third, extensive practice would incur smaller changes in the structure of the skill to adapt to the predictable perturbation.

\section{Method}

\subsection{Sample}

Forty-five college students ( 24 males and 21 females) 20 to 30 years of age (mean $24.7 \pm 3.4$ ), without prior experience with the sequential coincident timing task participated in this experiment. All participants were self-reported as right handed and had normal or corrected-to-normal vision. The study was performed in accordance with the ethical standards established in the 1964 Declaration of Helsinki amended in 1989.

\subsection{Instrument and Task}

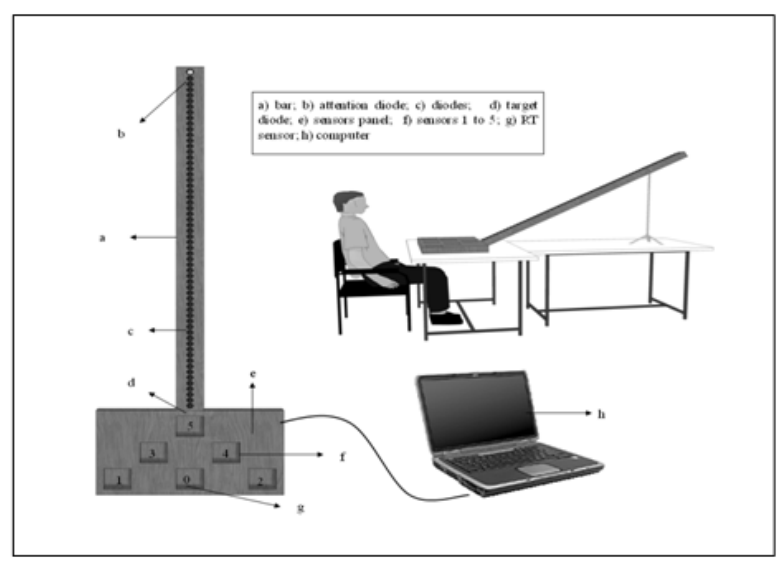

Figure 1. Instrument of the complex coincident timing task.
The apparatus was designed to accomplish a complex coincident timing task [2,7]. The participant sat in front of the table with the right hand on sensor 0 (Figure 1). The first LED (yellow diode) was turned on to attract the subject's attention, and when it turned off, all of the other red LEDs turned on and off in sequence, simulating an object descending the array toward the table.

The LEDs were turned on and off at a constant $1.104 \mathrm{~m} / \mathrm{s}$, which was the time constraint of the task. During this time, the participant had to touch the other five sensors in a pre-determined sequence $(1,4,2,3,5)$ at any velocity but with the constraint that the last sensor had to be touched at the same time that the last diode was turned on, i.e., coincident timing. We analyzed the task in five components: component 1 (C1) was the movement from sensor 0 (Reaction Time) to sensor 1; component 2 (C2) was the movement from sensor 1 to sensor 4 , and so on. Throughout the experiment, the participants could visually evaluate their touching the last sensor and the last diode turning on.

\subsection{Experiment Design}

The experiment was divided into two phases; the pre-exposure and the exposure phase. To investigate the first hypothesis, during the first phase of the experiment, three groups with three levels of performance stabilization were created: a) a pre-stabilization group (PG), with 10 trials (i.e., far too few trials for any participant to actually learn the task); b) a stabilization group (SG), whose participants had to perform three trials in a row with the absolute error less than or equal to $|30 \mathrm{msec}|$ in a maximum of 120 trials; and c) an extensive practice group (EG), whose participants had to perform six blocks of three trials in a row with absolute error less than or equal to $|30 \mathrm{msec}|$ in a maximum of 300 trials. These criteria were adopted because stability is slightly different in a continuous than in a discrete task [11], and coincident timing tasks are generally considered successful when the absolute error ranges from 1 to $30 \mathrm{msec}[12,13]$. This procedure guaranteed that the groups had different levels of performance stabilization [2,3] and that SG and EG had learned the task [14]. One volunteer did not reach the required performance level and was not included in data analysis.

The perceptual perturbation (change in the time constraint) was inserted three minutes after the end of the pre-exposure phase by manipulating the velocity at which the diodes were turned on and off. Although the velocity was constant during the first phase, during the second phase it was slower in the first half (the first 49 diodes) and faster during the second half (the last 50 diodes). The total time was the same as that in the previous phase, similarly to the method used by Fonseca et al. [2]; however, the perturbation was inserted in a predictable way, similarly to the Kagerer et al. study [10]. Different velocities of the time constraint were tested [3], and the easiest and the most difficult were adopted for the pre-exposure and exposure phases, respectively. The exposure phase comprised 25 trials with the same response sequence for touching the sensors as in the pre-exposure phase. 


\subsection{Data Analyzes}

The following dependent variables were used to assess performance: a) absolute error (AE), a measure to assess performance accuracy; b) variable error (VE), a measure to assess performance consistency; c) standard deviation of the relative time (RT) of the five components of the task, defined as the variability of the time spent to perform the movement from one sensor to another divided by the total movement time, which reflects the structure that controls the skill [15].

The results related to accuracy (AE) and consistency (VE) of performance during the pre-exposure phase were tested with a two-way ANOVA (3 groups $\mathrm{x} 2$ blocks) for the first and last blocks because the participants had different amounts of practice during the pre-exposure phase. During the exposure phase, data were analyzed with a two-way ANOVA (3 groups x 5 blocks) on blocks of five trials each. When necessary, a post hoc TukeyHSD test was adopted for pairwise comparison in both phases. The stability of the structure of the skill (relative time - RT) was tested with a MANOVA comparing the first and last blocks in both phases of the experiment, followed by a univariate test of significance when necessary. The type I error was set at $5 \%$, which means that the effects were significant at $\mathrm{p}<.05$. All data analysis was carried out using Statistica 8.0 for Windows.

\section{Results}

\subsection{Pre-Exposure Phase}

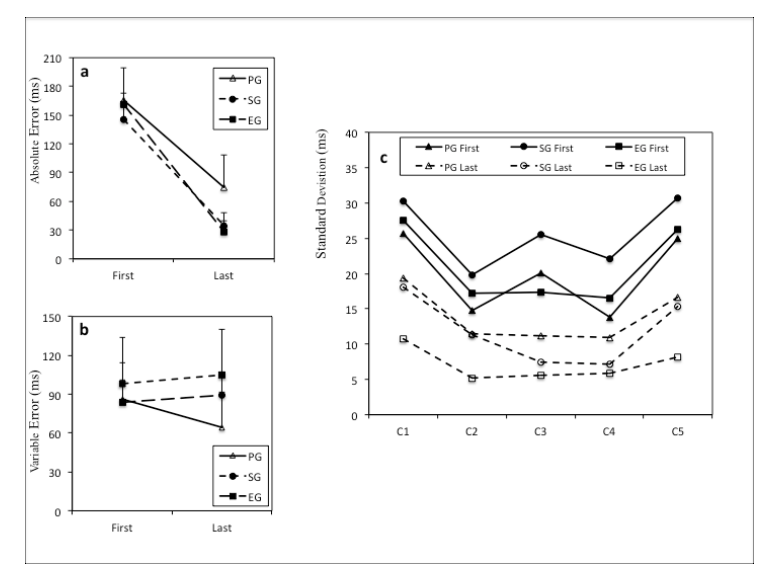

Figure 2. Comparison during the pre-exposure phase. The absolute error $(+S D)$ in the FIRST and LAST blocks of the pre-exposure phase (a). The variable error $(+S D)$ in the FIRST and LAST blocks of the pre-exposure phase (b). The standard deviation of the components in the FIRST and LAST blocks of the pre-exposure phase (c).

Data obtained in a previous study using an unpredictable perceptual perturbation [2] showed that extensive practice resulted in better performance (i.e., adaptation) than practice until performance stabilization. Moreover, extensive practice resulted in a smaller number of changes in the structure of the skill. In our study, Figure 1a shows that during the pre-exposure phase, there was a significant effect of practice on performance accuracy - AE diminished - $(\mathrm{F}$ $(1,42)=53.002, \mathrm{p}<0.001)$, and there was a significant difference between groups $(\mathrm{F}(2,42)=4.271, \mathrm{p}<0.020)$. The Tukey test determined that PG was less accurate than SG $(p<0.046)$ and EG ( $p<0.034)$. Figure $1 b$ shows a significant interaction between groups and blocks in the performance consistency measure $(\mathrm{F}(2,42)=3.038$, $\mathrm{p}<$ 0.038). The Tukey test determined that PG and SG became more consistent from the first to the last block $(\mathrm{p}<0.05)$, but EG was less consistent than PG $(\mathrm{p}<0.002)$ and SG $(\mathrm{p}<$ 0.023 ) in the last block.

Figure 1c shows that the RT of all three groups becomes more consistent from the first to the last block of this phase (MANOVA: Wilks' $\lambda=0.868, \mathrm{~F}(10,76)=11.246, \mathrm{p}<0.001$ ). Univariate tests showed that PG increased the consistency of components $\mathrm{C} 1$ and $\mathrm{C} 3$ (p < 0.01) and that both SG and EG increased consistency from $\mathrm{C} 1$ to $\mathrm{C} 5$ ( $\mathrm{p}<0.003)$.

\subsection{Exposure Phase}

Figure 2a shows significant interaction between groups and blocks during the exposure phase in performance accuracy $(\mathrm{F}(8,168)=6.453, \mathrm{p}<0.05)$. The Tukey test detected that in the first block, SG and EG were more accurate than PG ( $p<0.04$ and $p<0.001$, respectively) and that PG increased its accuracy from the first to the later blocks $(p<0.04)$. After the second block, all groups showed similar accuracy $(\mathrm{p}>0.05)$. Figure $2 \mathrm{~b}$ also shows significant interaction between groups and blocks in performance consistency $(\mathrm{F}(8,168)=2.164$, p < 0.032). The Tukey test detected that in the first block, EG was more consistent than PG ( $<<0.002)$ and SG $(p<0.018)$. Subsequently, EG consistency decreased in the other four blocks $(\mathrm{p}<0.05)$. Figure $2 \mathrm{c}$ shows that the consistency of the RT of the three groups changed from the first to the last block of the exposure phase (MANOVA: Wilks' $\lambda=0.173$, $\mathrm{F}(10,76)=12.602, \mathrm{p}<0.005)$. Univariate tests showed that PG diminished consistency of component $\mathrm{C} 1(\mathrm{p}<0.05)$ and increased consistency of component C3 (p < 0.05); SG increased consistency of component $\mathrm{C} 3(\mathrm{p}<0.05)$; and EG increased consistency of components C3 and C5 (p < 0.04).

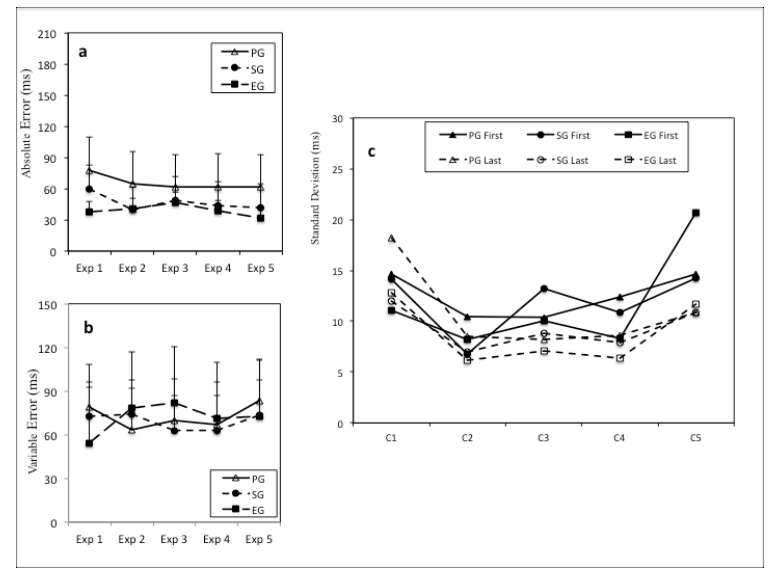

Figure 3. Comparison during the exposure phase. The absolute error $(+S D)$ in the Perturbation blocks of the exposure phase (a). The variable error $(+$ $\mathrm{SD})$ in the Perturbation blocks of the exposure phase (b). The standard deviation of the components of the Perturbation blocks of the exposure phase (c). 


\section{Discussion}

This study aimed to investigate whether extensive practice facilitates the acquisition of the ability to adapt to predictable perturbation in a sequential motor skill task compared to practice until performance stabilization or no stabilization at all. Initially, we analyzed the first phase of the experiment, and then we used the exposure phase to test our hypotheses. In the condition without perturbation, SG and EG had better performance accuracy than PG, which was reflected in the variability of the structure of the skill: although PG exhibited diminished variability in components $\mathrm{C} 1$ and $\mathrm{C} 3, \mathrm{SG}$ and EG exhibited diminished variability in all components. Similarly to the results from previous studies [2,3], practice until achieving a stabilization criterion seems to be enough to form a structure to control the skill in a coincident timing task. Moreover, at the end of this phase, PG and SG had higher performance consistency than EG. At first glance, this result may seem counterintuitive because it might be expected that performance variability would diminish with learning [16]. Nevertheless, there is a study showing that when practice extends beyond performance stabilization, the variability increases [3], most likely because changes in variability are related to the level of learning [17]. When a subject had only a few trials of practice (PG), performance accuracy was poor, but the participants did not know how to improve it. As a consequence, variability was low. With practice beyond performance stabilization (EG), it was possible to have high variability in performance while keeping the structure consistent at the same time. The results from the first phase indicate that low performance consistency resulting from extensive practice is helpful for adaptation [2,3]. These results led us to the first and second hypotheses.

The first hypothesis proposed that under predictable perturbation, extensive practice as well as practice until performance stabilization would produce similar performance and both would produce better performance than no performance stabilization at all. When all groups were exposed to predictable perturbation, the performance accuracy results showed that in the first block of the exposure phase, PG was less accurate than SG and EG; however, from the second block onward, all groups became similar. These results are different from those of other studies [2,3]. Most likely, the type of perturbation influenced these results. Ugrinowitsch et al. [3] manipulated both the time constraint and the sequence of movements, which made perturbation more difficult to adapt to than in our study. Fonseca et al. [2] manipulated the perturbation in an unpredictable way, whereas our study manipulated the perturbation in a predictable way. Predictable perturbation facilitates the ability of all groups to plan the whole sequence of movements before the movement onset [9]. In contrast, when the perturbation is unpredictable, changes in the plan of action can happen only after the movement onset, making it more difficult to change the movement and accomplish the goal of the task [8]. Although Fonseca et al.
[2] found better adaptation with extensive practice, in this study, SG and EG reached similar performance accuracy, showing that when it is possible to plan the movement before the movement onset, extensive practice is not necessary for performance adaptation. Moreover, PG, the group that practiced only 10 trials before facing the perturbation, reached similar performance accuracy in the second block of the exposure phase, giving support to the proposal that an unpredictable perturbation is more difficult to adapt to than a predictable one [9].

The second hypothesis proposed that the performance variability with extensive practice would diminish under the perturbation condition. Unexpectedly, the results from the variable error showed that EG had higher variability (lower consistency) than PG and SG prior to the perturbation. Most likely, this effect occurs because the stability of the system is related to the performance consistency when facing a perturbation [18]. This finding is another counterintuitive result because traditionally, it is expected that variability would decrease throughout the learning process [16]. However, when the perturbation was introduced, EG became more consistent than the other groups. Extensive practice increases performance variability, which is characteristic of the motor system [19] and can be useful for adaptation [20,21,22]. Reaching this level of performance (i.e., extensive practice) gives more information to the motor system, which constitutes a source of adaptability, a reserve capacity that allows a system to face perturbations [23]. Latash calls this reserve "abundance" [24]. During the first phase, PG had smaller variability than EG, but when it was exposed to perturbation, its variability was higher than that of EG, and its accuracy was lower. In other words, our results show that variability before performance stabilization is detrimental [16], particularly for adaptation, but after performance stabilization, variability is helpful for adaptation [21,22]. Moreover, our results show that variability is necessary for change [10] because stability and variability can have different mechanisms of control [25]. Other studies also showed that variability changes throughout the learning process [17] but did not analyze variability in an extensive practice condition.

The third hypothesis proposed that extensive practice would have a more stable structure and consequently would need smaller changes to adapt to the predictable perturbation. Accuracy and consistency of performance result from the structure of skill $[2,15]$. Relative time represents a structure of the skill showing the relationship between components of the task and tends to become fairly consistent but not rigid with practice [26]. This measure aims to identify changes in the relationship between components of the skill [27] by comparing the blocks immediately prior to with those during exposure to the perturbation. This analysis contributes to the understanding of how the structure of the skill behaves facing the perturbation, and it shows that the groups adapt differently to the perturbation. Although the perturbation was predictable, and all groups could plan the strategy before the start of the task, only PG exhibited diminished 
consistency in the structure, particularly in $\mathrm{C} 1$, indicating that the amount of practice during the pre-exposure phase was not enough for this group to acquire a flexible structure to control the skill. Moreover, this observation indicates that PG was using online planning, as $\mathrm{C} 1$ is the first component of the task. In addition, PG still increased the consistency of C3, similarly to SG and EG. However, SG and EG differed from $\mathrm{PG}$ in that they did not lose consistency in any component and became more consistent in components $\mathrm{C} 3$ and $\mathrm{C} 5$. These results indicate a hierarchy in managing the perturbation: $\mathrm{PG}$ exhibited increased consistency of one component and decreased consistency of another; SG exhibited increased consistency of one component; and EG exhibited increased consistency of two components.

Comparison with other studies using the same task indicates that predictable perceptual perturbation is easier to adapt to than predictable perceptual-motor perturbation because in the latter, SG adapts only in the last block with perturbation [3]. Moreover, predictable perturbations are easier to adapt to than unpredictable perturbations, as Fonseca et al. [2] found that SG exhibited decreased consistency in component C5. Our current results confirm the results of Richter et al. [9]. Planning a new movement to address the perturbation before the movement onset was not a great challenge for SG and EG, and these groups increased the consistency of the components even when exposed to perturbations. These differences in the structure are responsible for the differences in performance: although SG and EG had similar accuracy in the last block of the pre-exposure phase as well as in the first block of the exposure phase, EG was less consistent in pre-exposure but more consistent in the first block of exposure than SG. Practice beyond performance stabilization strengthens the structure of the skill, which can be verified by inserting the perturbation.

Because variability has been used to indicate the ability to adapt $[21,23,28,29]$, we analyzed the correlation between changes in performance accuracy $(\Delta)$ with the variability of the structure of the skill at the moment a perturbation was inserted. This analysis should indicate whether the variability of the structure is related to the ability to adapt. We used both variability measures (performance and relative time) in the block immediately before perturbation and the amount of change in performance accuracy $(\Delta)$ as a result of the perturbation. The tests showed a correlation between $\Delta$ and performance variability $(r=.63$ and $p<.012)$ for PG. There was no significant correlation for SG ( $p>.05)$, and there was a significant high correlation between $\Delta$ and variability of the structure $(r=.74$ and $p<.05)$ for EG. On one hand, these results show that the correlation of PG cannot be a predictor of adaptation because the small $\Delta$ in performance accuracy was related to high variability resulting from error [30]. On the other hand, the high correlation of EG results from the highest level of learning that can be a predictor of the ability to adapt. These results indicate that motor learning extends beyond the autonomous stage [5,31], as proposed by Tani [4] and Zanone and Kelso [32] and extend the results of Corrêa et al. [7], Ugrinowitsch et al. [3] and Fonseca et al. [2], confirming not only that performance increases with extensive practice but also that variability is functional when practice continues beyond performance stabilization. Most notably, we think that this is the first study to investigate extensive practice and predictable perturbation. Future work should compare the predictability of the perturbation in similar experimental conditions.

In conclusion, the results of the present study suggest that extensive practice provides a more flexible structure of the skill, which implies variability. However, the variability diminishes when adapting to predictable perceptual perturbation. Moreover, the results indicate that the small variability that remains after performance stabilization (EG) facilitates adaptation, indicating that motor learning is a continuous process that extends beyond performance stabilization.

\section{References}

[1] R.A. Scheidt, J.B. Dingwell, F.A. Mussa-Ivaldi, Learning to move amid uncertainty, J. Neurophys. vol. 86, pp. 971-985, 2001.

[2] F.S. Fonseca, R.N. Benda, V.L.S. Profeta, H. Ugrinowitsch, Extensive Practice Improves Adaptation to Unpredictable Perturbations in a Sequential Coincident Timing Task. Neurosci. Lett. vol. 517, pp. 123-127, 2012.

[3] H. Ugrinowitsch, S.P. Santos-Naves, M.V. Carbinatto, R.N. Benda, G. Tani, Motor skill adaptation depends on the level of learning, Inter. J. Hum. Soc. Scie. vol. 6, pp. 177-181, 2011.

[4] Tani, G.; Corrêa, U. C.; Basso, L.; Benda, R.N.; Ugrinowitsch, H.; Choshi, K. An Adaptive Process Model of Motor Learning: Insights for the Teaching of Motor Skills. Nonlin. Dynam. Psych. Life Sci., vol. 18, pp. 47-66, 2014.

[5] P.M. Fitts, M.I. Posner, Human Performance. Belmont: Brooks-Cole, 1967, 162 pp.

[6] R.W. Pew, Acquisition of hierarchical control over the temporal organization of skill, J. Exp. Psych. vol. 71, pp. 764-761, 1966.

[7] U.C. Corrêa, H. Ugrinowitsch, R.N. Benda, G. Tani, Effects of practice schedule on the adaptive process of motor learning. Rev. Port. Cien. Desp. vol. 10, pp. 158-171, 2010.

[8] S. Richter, J. Konczak, M. Maschke, T. Kalenscher, D. Timmann, A.R. Illenberger, A.R.I.K.T. Kalveram, Adaptive motor behavior of cerebellar patients during exposure to unfamiliar external forces. J. Mot. Behav. vol. 36, pp. 28-38, 2004a.

[9] S. Richter, P. Jansen-Osmann, J. Konczak, K. Kalveram, Motor adaptation to different dynamic environments is facilitated by indicative context stimuli, Psych. Res. vol. 68 pp. 245-251, 2004b.

[10] F.A. Kagerer, J.L. Contreras-Vidal, G.E. Stelmach, Adaptation to gradual as compared with sudden visou-motor distortions, Exp. Brain Res. vol. 115, pp. 557-561, 1997.

[11] D. Sternard, M.O. Abe, Variability, noise, and sensitivity to error in motor learning a motor task, in: F. Danion, M.L. Latash, (Eds.), Motor Control: Theories, Experiments, and Applications, Oxford, University Press, 2011, pp. 267-294. 
[12] P.W. Dorfman, Timing and anticipation: a developmental perspective. J. Mot. Behav. vol. 9, pp. 67-79, 1977.

[13] L.R.T. Willians, J.M. Jasiewicz, R.W. Simmons, Coincidence timing of finger, arm, and whole body movements. Percep. and Mot. Skills, vol. 92, pp. 535-547, 2000.

[14] N. Bernstein, The Co-ordination and Regulation of Human Movements. Oxford, Pergamon, 1967, 196 pp.

[15] K.M. Newell, Change in movement and skill: learning, retention and transfer, in: M. Latash, M. Turvey (Eds.), Dexterity and Movement, Lawrence Erlbaum, New Jersey, 1996, pp. 393-430.

[16] R.A. Schmidt, A schema theory of discrete motor skill learning. Psych. Rev. vol. 82, pp. 225-260, 1975.

[17] L. Li, J.M. Haddad, J. Hamil, Stability and variability may respond differently to changes in walking speed. Hum. Mov. Sci. vol. 24, pp. 257-267, 2005.

[18] E. Burdet, K.P. Tee, I. Mareels, T.E. Milner, C.M. Chew, D.W. Franklin, R. Osu, M. Kawato, Stability and motor adaptation in human arm movements. Biol. Cyber. vol. 94, pp. 20-32, 2006.

[19] K.M. Newell, D.M. Corcos, Variability and Motor Control. Champaign, Illions, Human Kinetics, 1993, 510 p.

[20] M.A. Riley, M.T. Turvey, Variability and determinism in motor behavior. J. Mot. Behav. vol. 34, pp. 94-125, 2002.

[21] K. Torre, R. Balasubramaniam, Disentangling stability, variability and adaptability in human performance: Focus on the interplay between local variance and serial correlation. J. Exp. Psych.: Hum. Perc. Perf. vol. 37, pp. 539-550, 2011.

[22] R.E. van Emmerik, E.E.H. van Wegen, On the functional aspects of variability in postural control. Exerc. Sport Sci. Rev. vol. 30, pp. 177-193, 2002.

[23] G. Tani, Processo adaptativo em aprendizagem motora: o papel da Variabilidade. [Adaptive process and motor learning: the role of variability]. Revista Paulista de Educação Física, [Paulista J. Phys. Educ.] vol. 14, pp. 55-61, 2000.

[24] M.L. Latash, F. Danion, J.F. Scholz, V.M. Zatiorsky, G. Schöner, Approaches to analysis of handwriting as a task of coordinating a redundant motor system. Hum. Mov. Sci. vol. 22, pp. 153-171, 2003.

[25] J.P. Scholz, G. Schöner, The uncontrolled manifold concept: Identifying control variables for a functional task. Exp. Brain Res. vol. 126, pp. 289-306, 1999.

[26] H. Heuer, Invariant relative timing in motor-program theory. In: J. Fagard, P.H. Wolff, (Eds.) The development of timing control and temporal organization in coordinated-actions. North-Holland, Amsterdan, 1991, pp. 37-68.

[27] E.J. Manoel, L. Basso, U.C. Correa, G. Tani, Modularity and hierarchical organization of action programs in human acquisition of graphic skills, Neurosci. Lett. vol. 335, pp. 83-86, 2002.

[28] J.A.S. Kelso, Dynamic Patterns, the Self-Organization of Brain and Behavior. Cambridge, MA: MIT Press, 1995, 334 p.

[29] A.B. Slifkin, K.M. Newell, Noise, information transmission, and force variability. J. Exp. Psych.: Hum. Perc. Perf. vol. 25, pp. 837-851, 1999.

[30] E.J. Manoel, K.J. Connolly, Variability and the development of skill actions, Intern. J. Psychophys. vol. 19, pp. 129-147, 1995.

[31] A.M. Gentile, A working model of skill acquisition with application to teaching. Quest, vol. 17, pp. 3-23, 1972.

[32] P.G. Zanone, J.A.S. Kelso, Learning and transfer as dynamical paradigms for behavioral change. In: G.E. Stelmach, J. Requin (Eds.) Tutorials in Motor Behavior II. Amsterdam, Elsevier Science, 1992, pp.563-582. 